\title{
The protective effect of activating Nrf2 / HO-1 signaling pathway on cardiomyocyte apoptosis after coronary microembolization in rats
}

Jiabao Liang, Lang Li ${ }^{*}$ D, Yuhan Sun, Wenkai He, Xiantao Wang and Qiang Su

\begin{abstract}
Background: Myocardial apoptosis is closely related to myocardial injury caused by coronary microembolization (CME).Nuclear factor erythroid 2-like (Nrf2) has been taken into account as an inhibitor of apoptosis in various tissues. Thus, this research aims to investigate which part Nrf2/HO-1 signaling pathway plays in myocardial apoptosis process following the effect of CME on rats.

Methods: Separate 40 rats then form them into a group of shame, a group of CME, a group of CME plus AAVNrf2(AAV-Nrf2 (CME) group) and a group of CME plus AAV-control (AAV-control (CME) group) stochastically and averagely. Rat CME was established by injecting into the left ventricular chamber, with or without pretreatment of adeno-associated virus Nrf2 (AAV-Nrf2). Echocardiological measurements, using Terminal-deoxynucleoitidyl Transferase Mediated Nick End Labeling (TUNEL) to stain, conducting Quantitative PCR in real time (RT-PCR) as well as Western blotting to evaluate the impacts of them functionally, morphologically and molecularly in CME.

Results: Nrf2 decreased in cardiomyocytes after CME. Upregulation of Nrf2 inside an organism through AAV connect to improving the function of heart as well as attenuating myocardial apoptosis, following the restrain of proapoptotic mRNAs and proteins like caspase-3, caspase-9 and bax expressing as well as the increase of antiapoptotic mRNA and proteins like $\mathrm{HO}-1$ and bcl-2 expressing.
\end{abstract}

Conclusion: Activation of Nrf2/HO-1 pathway can improve CME-induced cardiac dysfunction effectively and also reduce the myocardial apoptosis.

Keywords: Nuclear factor erythroid 2-like, Heme oxygenase-1, Coronary microembolization, Apoptosis

\section{Background}

Coronary microembolization (CME) is among those vital causes of dysfunctional heart without atherosclerotic obstacle inside the epicardial coronary artery. Tens of years ago, CME was discovered at postmortem for the first time in patients who died as a result of a unexpected cardiac events [1]. Nevertheless, CME, following thrombolytic treatment and coronary disturbances for patients suffering a coronary thrombus, is becoming more regularly [2], or a remote arteriole thrombus in a spontaneous lysis. CME may cause myocardial perfusion-contraction mismatch,

\footnotetext{
* Correspondence: drlilang@163.com

Department of Cardiology, The First Affiliated Hospital of Guangxi Medical University, Nanning, Guangxi Zhuang Autonomous Region 530021, China
}

arrhythmias, inflammation and micro-infarction and so on, which can possibly result in harmful or fatal consequences [3-8]. CME is a fundamental reason of nonblocked coronary artery illness. Numerous investigations have evidenced the underlying mechanisms of CMEinduced myocardial damage as well as relieve the deleterious effects of CME. A considerable amount of studies demonstrated that cardiomyocyte apoptosis was a vital part of the mechanism of CME-induced myocardial damage [9-11], and extra modulatory mechanisms still need to be explained because of the overall complexity of myocardial injury.

Heme oxygenase- $1(\mathrm{HO}-1)$ is a phaselIdefense enzyme which has potent antiapoptotic as well as antioxidative 
stress effects. In those HT22 cells, HO-1 is over expressed which confer antiapoptotic protective effects against H/R [12]. Nuclear factor erythroid 2-like (Nrf2) is a primary element of transcription. It adjusts numerous ways of apoptosis in a cell [13]. Recent studies have paid more attention to the Nrf2/HO-1 approach that is a key part in the apoptosis and that regulates and lowers the cardiomyocyte apoptosis [14].

The purpose of the current research was in order to determine the part of Nrf2/HO-1 in CME-induced myocardial apoptosis. Here, we focused on the interactions of Nrf2/HO-1 pathway with the bcl-2, bax, caspase 3 and caspase 9 in CME-induced myocardial apoptosis. The study suggested that $\mathrm{Nrf} 2 / \mathrm{HO}-1$ was a major part in CME-induced myocardial apoptosis and activation of Nrf2/HO-1 pathway could remarkably restrain apoptosis in CME-induced myocardial as well as enhance the function of heart. We supplied a possible CME-induced myocardial apoptosis mechanism.

\section{Methods}

\section{Animal preparation}

Sprague-Dawley rats (250-300 g) were gained from the Animal Center of the Guangxi Medical Collage which is in Nanning, China. During every phase of this experiment, the rats were raised with manipulated light status, moisture, with clean water and feed provided unlimited. All the procedures were authorized and supervised by the Animal Experiment Ethics Committees of Guangxi Medical Collage.

\section{Design, synthesis as well as transfection of AAV-Nrf2}

The adeno-associated virus Nrf2(AAV-Nrf2) gene sequence was located in GeneBank (Gene ID: 83619) and compounded by Hanbio Biotechnology Co (Shanghai, China). Control AAV was also bought from this company. A dose of $10 \times 11 \mathrm{E}$ in all injected by tail veins was mixed with saline for each rat.

\section{Creation of a CME model and grouping}

CME model was generated as described by $\mathrm{Li} \mathrm{L}$ et al. previously [15]. Forty rats were separated then formed into 4 groups, containing a sham group, CME group, CME plus AAV-Nrf2 group (AAV-Nrf2(CME) group) and CME plus AAV-control group (AAV-control (CME) group), with 10 rats in each one of them. AAV-Nrf2(CME) group and AAV-control (CME) group both received 2-week transfection of AAV-Nrf2 or AAV-control virus. And then, the two groups received the following operation: put simply, a sternotomy in the middle the second and third intercostal was undertaken. The pericardium was unfolded and the increasing aorta was totally revealed. The plastic microspheres (3000 microspheres per rat, diameter of
$42 \mu \mathrm{m}$, Biosphere Medical Inc., Rockland, USA) dissolved in $0.1 \mathrm{~mL}$ NS were injected into the ventricular (LV) chamber on the left and meanwhile clamping the ascending aorta with the use of the hemostatic forceps during the $10 \mathrm{~s}$. Rats in the control group received $0.1 \mathrm{ml}$ normal saline (PubChem CID: 5234) merely as the sham group did.

\section{Echocardiography study}

The left ventricle was detected applying echocardiography which was used to examine the left ventricle of rats, which were lightly anesthetized by injecting $10 \%$ chloral hydrate $(1-2 \mathrm{ml} / \mathrm{kg})$ into an enterocoelia. In a word, an S12 transducer was used to be put on the left side and anterior position of chest wall to gain the left ventricle end-diastolic dimension (LVEDd), left ventricle ejection fraction (LVEF), cardiac output (CO) as well as fractional shortening (FS). A Philips Sonos 7500 system (Philips, Andover, USA) was used then all echocardiography was carried on by a veteran physician. Evaluating cardiac function three times, and the average values of the three figures were taken.

\section{Myocardial micro-infarct size measurement}

HBFP stain was applied to detect myocardial ischemia in an early stage or infarct area. The dyed yellow or brown myocardial tissue was normal while the red tissue was ischemia or necrotic. A DMR-Q550 pathological picture analyzer (Lei, Germany) analyzed The HBFP-stained sections before. In short, 10 optical areas in the microscope (magnification, $\times 100$ ) were casually selected from every part and Leica Qwin analysis software was used to observe. The formula- ischemic area/total area $\times 100 \%$ was used to calculate the relative ischemic area [16].

\section{Using TUNEL assay to examining myocardial apoptosis}

This process was grimly conformed to the instructions of Kit specification. Apoptotic nuclei showed yellowbrown while the normal colour is light blue (TUNEL positive), and a whole amount of 10 non-overlapping areas (magnification, $\times 400$ ) from every slice which was observed stochastically [17]. The myocardial apoptotic ratio $=$ apoptotic number/entire cell number $\times 100 \%$ was to be used.

\section{Quantitative Polymerase Chain Reaction in real time (RT- PCR)}

We extracted the entire RNA from the cardiac tissue of rats with TRIzol ${ }^{\circ}$ Reagent (Takara, Japan) $6 \mathrm{~h}$ after CME operation. Reverse transcription RNA $(1 \mu \mathrm{g})$ was performed applying a reverse transcriptase kit (Takara, Japan) referring to the instructions provided by the manufacturer. Reactions involved $2 \mu \mathrm{L}$ cDNA, $2 \mu \mathrm{L}$ primers and $12.5 \mu \mathrm{L}$ SYBR Premix Ex Taq II, and put 
into a ultimate volume of $20 \mu \mathrm{L}$ of water. Real time-PCR was undertaken the next phase situations: ten-min initial denaturation at $95 \mathrm{C}$ following $15-\mathrm{s} 40$ phases of amplification with $95 \mathrm{C}$ as well as 1 -min $60{ }^{\circ} \mathrm{C}$. PCR in real time was applied with the following primers: $5^{\prime}$ GCTGCCATTAGTCAGTCGCTCTC $-3^{\prime}$ and $5^{\prime}-$ ACC GTGCCTTCAGTGTGCTTC-3' for the rat nuclear relative factor 2-like (Nrf2), 5'- TTAAGCTGGTGATGG CCTCC $-3^{\prime}$ and $5^{\prime}$ - GTGGGGCATAGACTGGGTTC-3' for the rat heme oxygenase1(HO-1), and 5'-ACTGC TTCCCAGACCCACA-3' and 5'-CGAGACCTTGGAAC ACAGAGAA-3' for caspase9, and 5'-GCAGCAGCCTC AAATTGTTGAC- $3^{\prime}$ and $5^{\prime}$-TGCTCCGGCTCAAACCA TC-3' for caspase3, and 5' -AGACACCTGACCTTGGA-3 and 5'-TTGAAGTTGCCATCAGCAAACA-3 for bax, and 5'-AGACACCTGACCTTGGA-3 and 5'-TTGAAG TTGCCATCAGCAAACA-3 for bcl-2, and 5'-GAGATT ACTGCCCTGGCTCCTA-3' and 5'-CATCGTACTCCT GCTTGCTGAT-3' for $\beta$-actin. The dissociation curve was analyzed to confirm specific amplification. The amplification of the overall cDNA samples were divided into 3 portions and standardized compared to a triplicate of $\beta$-actin in this dish. The expression of the data was $2-\Delta \Delta \mathrm{Ct}$.

\section{Western blotting}

Proteins were extracted from tissue samples from $6 \mathrm{~h}$ after CME operation. Subsequently, the protein $(50 \mu \mathrm{g})$ was quantified using a BCA assay (Solarbio, Beijing, China) and was loaded onto a 10\% SDS-PAGE gel for electrophoresis and then transferred into a PVDF membrane (Millipore). Blocking with $5 \%$ non-fat milk in TBS at the temperature in a room for $1 \mathrm{~h}$, following the incubation of the membranes with a 1:500-10,000 dilution of primary antibody (anti-Nrf2, anti-HO-1, anti-caspase-3, anticaspase-9, anti-bax, as well as anti-bcl-2; Abcam, UK) in buffer overnight at $4{ }^{\circ} \mathrm{C}$, after washing 3 times in TBS, the
Table 1 The weights and heart rates of four groups before operation. There were not any statistically significant differences among four groups

\begin{tabular}{lllll}
\hline Groups & Sham & CME & AAV-Nrf2 & AAV-control \\
\hline Weight (g) & $251.2 \pm 10.3$ & $253.2 \pm 12.2$ & $249.9 \pm 11.3$ & $250.8 \pm 9.3$ \\
Heart rate (bpm) & $463 \pm 20$ & $460 \pm 27$ & $458 \pm 32$ & $462 \pm 24$ \\
\hline
\end{tabular}

Data is shown as Mean \pm SD

Nrf2 nuclear factor erythroid 2-like, $A A V$ adeno-associated virus

${ }^{a} P<0.05$ contrasted with sham

${ }^{\mathrm{b}} \mathrm{P}<0.05$ contrasted with CME or AAV control

incubation happened with a 1:10,000 dilution of fluorescent anti-rabbit secondary antibody (Abcam, UK) in buffer at the temperature in the room for $2 \mathrm{~h}$. The immunoreactive bands were observed by exposing the blots in an Odyssey infrared imaging system (LI-COR).

\section{ELISA}

To measure the expression of cTnI in serum, blood samples from each rat were collected for ELISA $6 \mathrm{~h}$ after CME operation based on the instructions provided by the manufacturer.

\section{Preparation of the tissue samples}

Ten rats per group were killed under anesthesia $6 \mathrm{~h}$ after CME operation. Blood samples were collected and used for enzyme linked immunoassay (ELISA). Heart tissue was harvested. Four tissue samples were fixed in $4 \%$ paraformaldehyde (PubChem CID: 62081) for $24 \mathrm{~h}$ for preparation of paraffin sections and the myocardial infarct size was detected by HE staining and hematoxylin-red complex picric acid (HBFP) staining. The apoptosis of myocardium was detected by TUNEL staining. Three hearts were preparation for Real time-PCR. The remaining heart tissue was used for the preparation of Western blotting. All tissue samples were prepared in an identical manner.
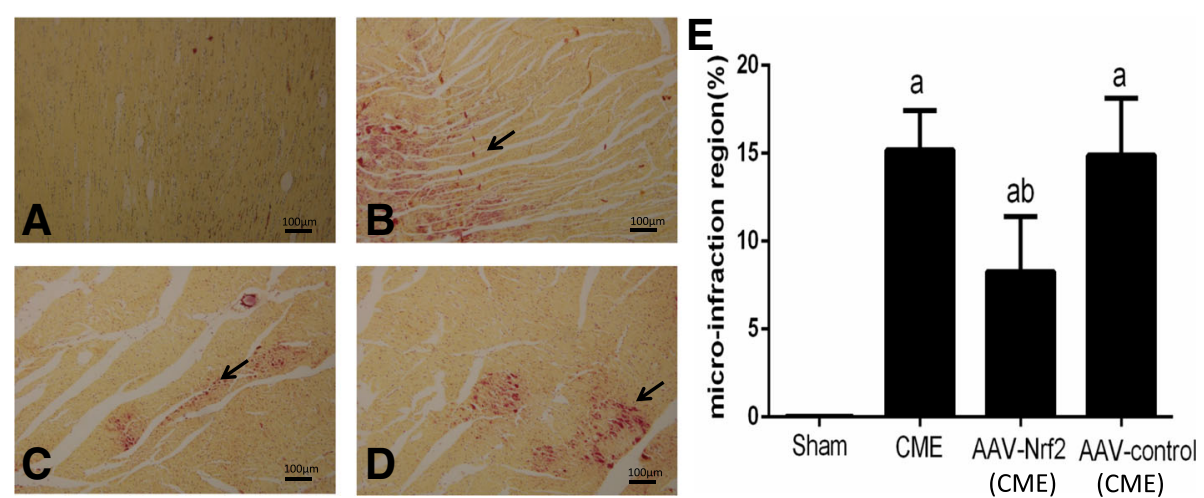

Fig. 1 Microinfarction detected with HBFP staining $(\times 100)$, bar $=100 \mu \mathrm{m}$. The normal myocardial staining was yellow, while the stain of ischemic myocardium was reddish. Arrows reveal microinfarction focus. a Sham; b CME; c AAV-Nrf2(CME); d AAV-control (CME). e The percentage of microinfarction area. AAV-Nrf2(CME): rats injected Nrf2 undergone CME operation; AAV-control (CME): rats injected AAV-control undergone CME operation; CME: coronary microembolization; Nrf 2: nuclear factor erythroid 2-like; AAV: adeno-associated virus. Data is shown as Mean \pm SD. a: $P<0.05$ contrasted with sham; b: $P<0.05$ contrasted with CME or AAV control (CME) 


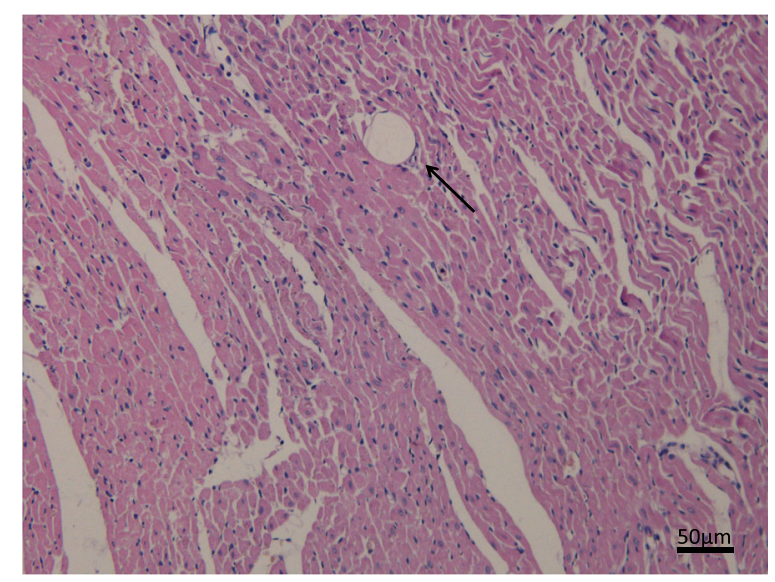

Fig. 2 Histopathology of post-CME myocardial microinfarcts $(\times 200)$, bar $=50 \mu \mathrm{m}$. Tissue samples' HE stain from the CME group. The arrow reveals the exist of a $42-\mu \mathrm{m}$ microsphere following CME

\section{Statistical analysis}

Data was analyzed by SPSS v18.0 (Chicago, Illinois, USA). Continuous variables are the mean \pm SD. We applied one-way ANOVA (Analysis of Variance) to contrast differences among groups. Categorical variables were reported as frequencies and percentages (\%), and differences between groups were examined by Fisher exact examination or chi-square examination. $P<0.05$ was considered statistically remarkable.

\section{Result}

\section{Groups of animal}

No significant discrepancies statistically $(P>0.05)$ in weight of the body or rate of the heart were testified among these four groups (Table 1).

\section{HBFP staining and HE staining}

Six hours after CME modeling, the HBFP staining showed that no obvious myocardial microinfarction in the sham group. Meanwhile, it was remarkably distinct in the CME group and AAV-control (CME) group $(P<0.05)$. As contrasted with $\mathrm{CME}$ group,
AAV-Nrf2 pretreatment remarkably reduced the micro-infarction area $(P<0.05)$. The proportions of micro-infraction area in the sham, CME, AAVNrf2(CME) as well as AAV-control (CME) were $0.03 \pm 0.01,15.20 \pm 2.23,8.28 \pm 3.12,14.90 \pm 3.22$ respectively (Fig. 1e). The HE staining showed that microspheres were injected into the myocardium (Fig. 2).

\section{AAV-Nrf2 pretreatment improved the function of heart following CME}

The effects of echocardiography represented that the CME group and the AAV-control (CME) group reduced the function of cardiac systolic comparing with the sham group significantly, as showed by the significantly decreased LVEF, FS, as well as CO $(P<0.05)$, while LEVDd was remarkably ascended $(P<0.05)$ at $6 \mathrm{~h}$ after $\mathrm{CME}$ modeling. Conversely, LVEF, FS, CO significantly increased while LEVDd was remarkably reduced in the AAV-Nrf2(CME) group $(P<0.05)$ (Table 2$)$.

\section{AAV-Nrf2 pretreatment attenuated myocardial damage following CME}

Six hours following CME modeling, serum levels of cTnI in rats from the sham group $(0.32 \pm 0.015 \mu \mathrm{g} / \mathrm{L}$ vs $0.05 \pm 0.002 \mu \mathrm{g} / \mathrm{L}, P<0.05)$ were lower than the CME group or the AAV-control (CME) group. What's more, AAV-Nrf2 pretreatment was involved with reducing cTnI levels $(0.19 \pm 0.014 \mu \mathrm{g} / \mathrm{L})$ when contrasted with CME group as well $(P<0.05)$.

\section{AAV-Nrf2 pretreatment decreased myocardial apoptosis following CME}

AAV-Nrf2 pretreatment reduced myocardial apoptosis after CME and myocardial apoptosis was measured by TUNEL staining. More TUNEL-positive (yellow-brown) cardiomyocytes can be examined in rats from the CME group when contrasted with the sham group, $(P<0.05)$. In addition, the AAV-Nrf2 remarkably decreased scale of apoptotic cells relatively after CME $(P<0.05)$. The proportions of myocardial apoptotic cells in the sham,

Table 2 Coefficient of the function of heart in rats in every group $6 \mathrm{~h}$ following CME modeling: the echocardiologic measurement conclusions

\begin{tabular}{lllll}
\hline Groups & LVEF (\%) & FS (\%) & LVEDd (mm) & CO (L/min) \\
\hline Sham & $89.25 \pm 2.52$ & $42.33 \pm 2.56$ & $5.67 \pm 2.12$ & $0.232 \pm 0.018$ \\
CME & $52.63 \pm 1.78^{\mathrm{a}}$ & $36.25 \pm 3.22^{\mathrm{a}}$ & $6.51 \pm 3.33^{\mathrm{a}}$ & $0.192 \pm 0.033^{\mathrm{a}}$ \\
AAV-Nrf2 (CME) & $70.44 \pm 3.21^{\mathrm{ab}}$ & $44.74 \pm 1.58^{\mathrm{ab}}$ & $5.79 \pm 1.66^{\mathrm{ab}}$ & $0.255 \pm 0.025^{\mathrm{ab}}$ \\
AAV-control (CME) & $52.87 \pm 2.12^{\mathrm{a}}$ & $34.55 \pm 2.24^{\mathrm{a}}$ & $6.48 \pm 1.85^{\mathrm{a}}$ & $0.201 \pm 0.011^{\mathrm{a}}$ \\
\hline
\end{tabular}

Data is shown as Mean $\pm \mathrm{SD}$

AAV-Nrf2(CME) rats injected Nrf2 undergone CME operation, AAV-control (CME) rats injected AAV-control undergone CME operation, CME coronary microembolization, $L V E F$ left ventricular ejection fraction, FS fractional shortening, LVEDd left ventricular end -diastolic diameter, CO cardiac output, Nrf2 nuclear factor erythroid 2-like, $A A V$ adeno-associated virus

a $P<0.05$ contrasted with sham

${ }^{\mathrm{b}} P<0.05$ contrasted with CME or AAV control (CME) 

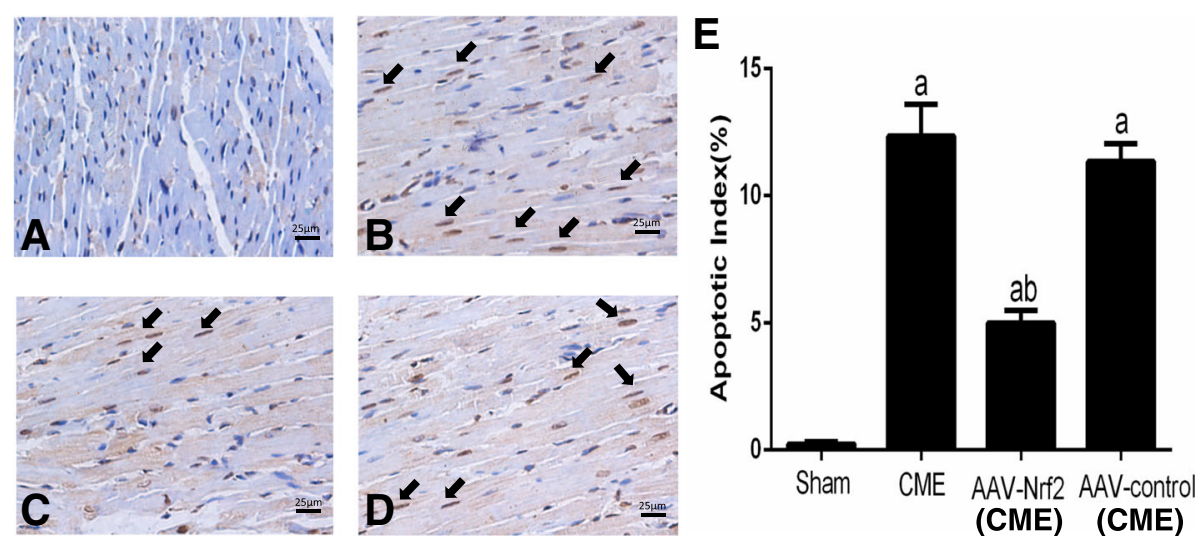

Fig. 3 Typical pictures and quantified analysis of myocardial apoptosis in rats from every $6 \mathrm{~h}$ after CME modeling: TUNEL staining conclusions. The staining of apoptotic cardiomyocyte nuclei (arrows) are brown $(\times 400)$, bar $=25 \mu \mathrm{m}$. Conclusions of quantified detection revealed that the apoptotic coefficients of myocardium were mainly higher in the CME or the control groups contrasted with those in Nrf2 groups. a Sham; b CME; c AAV$\mathrm{Nrf2(CME);} \mathrm{d} \mathrm{AAV-control} \mathrm{(CME);} \mathrm{e} \mathrm{the} \mathrm{histogram} \mathrm{of} \mathrm{the} \mathrm{apoptotic} \mathrm{index} \mathrm{of} \mathrm{each} \mathrm{group} \mathrm{of} \mathrm{rats.} \mathrm{AAV-Nrf2(CME):} \mathrm{rats} \mathrm{injected} \mathrm{Nrf2} \mathrm{undergone} \mathrm{CME} \mathrm{oper-}$ ation; AAV-control (CME): rats injected AAV-control undergone CME operation; CME: coronary microembolization; Nrf 2: nuclear factor erythroid 2-like; AAV: adeno-associated virus. Data is presented as Mean \pm SD a: $P<0.05$ contrasted with sham; $b: P<0.05$ compared with CME or AAV control (CME)

CME, AAV-Nrf2(CME) and AAV-control (CME) were $0.23 \pm 0.10,12.34 \pm 1.27,5.01 \pm 0.50,11.34 \pm 0.72$ respectively (Fig. 3e).

The mRNA levels of Nrf2, HO-1, bax, bcl-2, caspase3 and caspase9

The mRNA levels of Nrf2 in the sham group and the AAV-Nrf2(CME) group $(P<0.05)$ were remarkably higher than those in the CME group. Moreover, the
mRNA Nrf2 (Fig. 4a) level, HO-1 (Fig. 4d) and bcl-2 (Fig. 4e) were significantly lower in the CME and the AAV-control (CME) groups than those in the sham group and AAV-Nrf2(CME) group $(P<0.05)$. In addition, the mRNA levels of bax (Fig. 4b), caspase-3 (Fig. 4c) and caspase-9 (Fig. 4f) were increased significantly in the CME and the AAV-control (CME) groups contrasted with those in the sham group and the AAVNrf2(CME) group $(P<0.05)$.

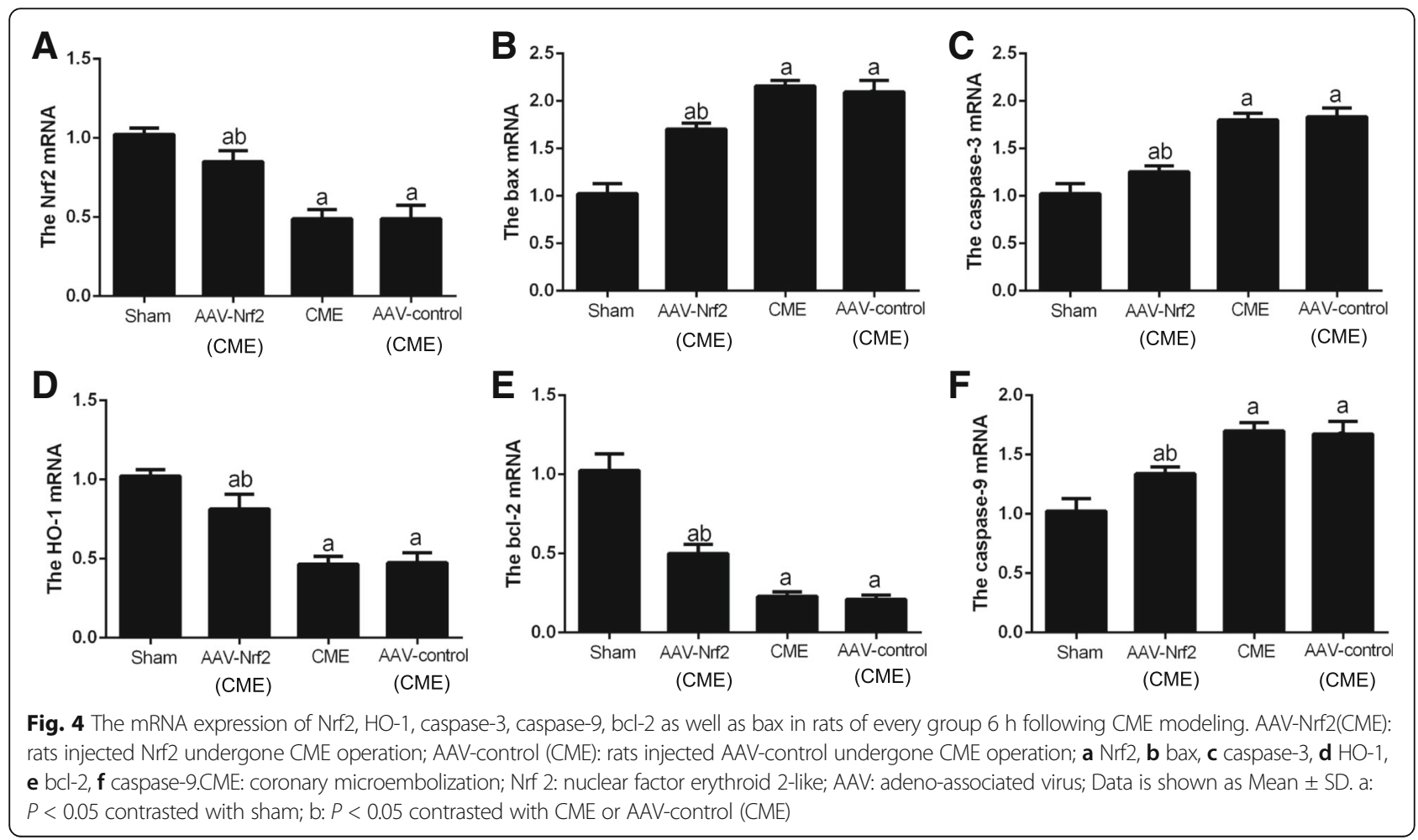




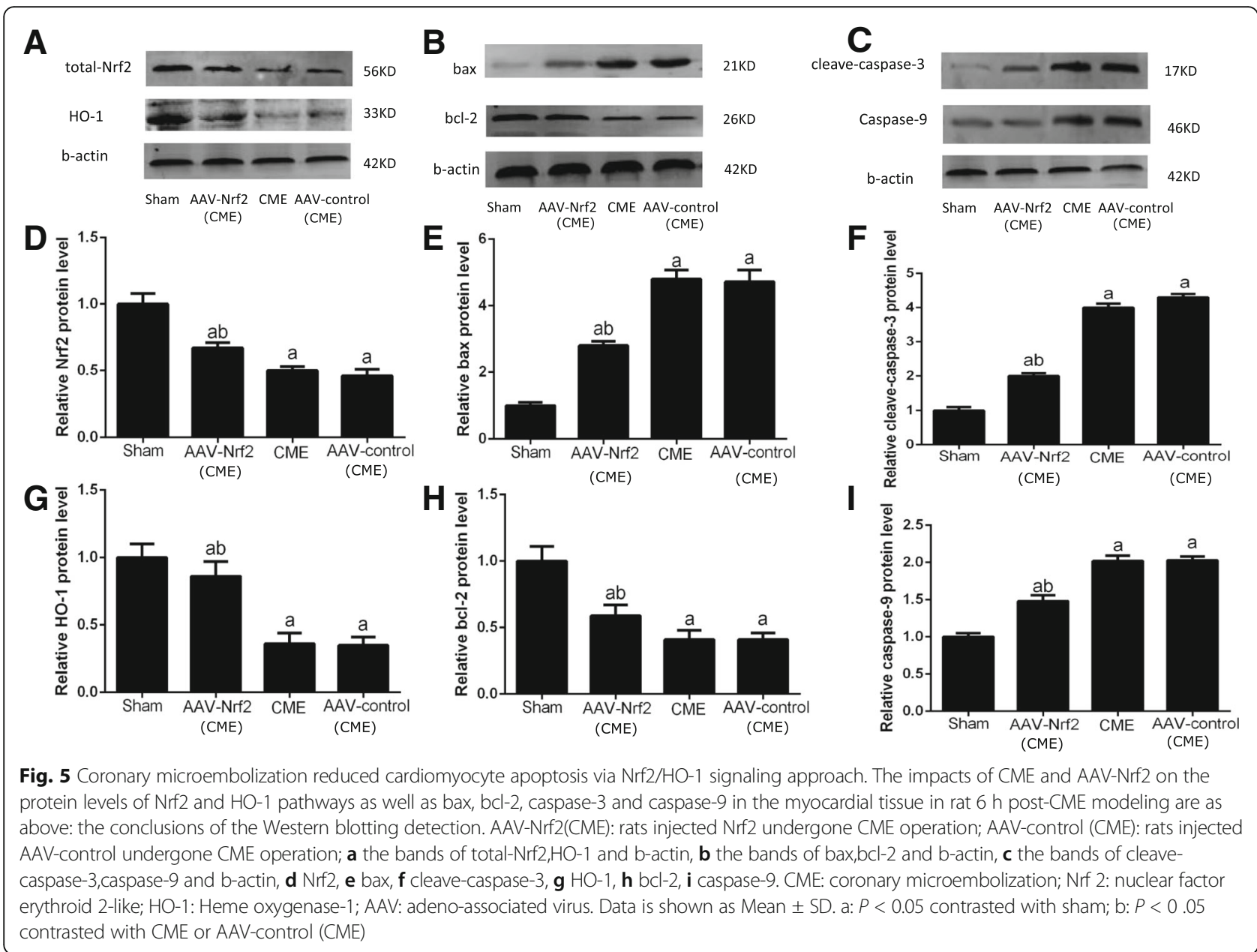

\section{AAV-Nrf2 pretreatment activated myocardial HO-1 signaling in $\mathrm{CME}$}

Western blotting exhibited significantly down-adjustment of HO-1 in CME and AAV-control (CME) groups contrasted with the sham group $(P<0.05)$. Nevertheless, AAV-Nrf2 pretreatment enhanced the expression of $\mathrm{HO}-1$ protein (Fig. 5g) and bcl-2 protein (Fig. 5h) contrasted with the CME group or the AAV-control (CME) group $(P<0.05)$, and reduced the expression of cleave-caspase-3 protein (Fig. 5f), caspase-9 protein (Fig. 5i) and bax protein (Fig. 5f) $(P<0.05)$.

\section{Discussion}

In our research, we testified that transfecting Nrf2 attenuated myocardial apoptosis and partially reversed CME, and that Nrf2/HO- 1 signaling pathway was concluded in the pathogenesis of CME-induced myocardial apoptosis was saw. The results of this research emphasized the significant part that $\mathrm{Nrf} 2 / \mathrm{HO}-1$ plays in the pathogenesis of CME-connected myocardial dysfunction as well as in the apoptosis. We initially illuminate the part of $\mathrm{Nrf} 2 / \mathrm{HO}-1$ pathway plays in the CME model. These findings could enable us to illustrate the mechanisms by which $\mathrm{Nrf} 2$ / HO-1 mediates myocardial injury supports our hypothesis that Nrf2 might represent a potential target for therapy.

$\mathrm{AAV}$ is a small and nonpathogenic human virus that belongs to the parvovirus family and was originally discovered in the mid-1960s. In the preliminary experiment, we found that there were no statistical difference of cleavecaspase-3 among the groups of rats transfected by AAV-Nrf2 and AAV-control of which rats were not undergone CME and the control rats (Fig. 6).AAV is as a contaminant of cell culture also infected with adenovirus. The high efficiency of in vivo transduction of postmitotic tissues, including heart, brain, and retina, combined with its low immunogenicity, led to the widespread use of AAV as a transfer method for gene therapy in various organs [18-20]. The results and previous studies can be tended to indicate that it has no effect on myocardial apoptosis. Therefore, the rats, which were not undergone CME, were transfected by AAV-Nrf2 and AAV-control groups were not included in further analyses.

Microcirculation of acute dysfunction and myocardial damage perfusion is a characteristic of CME. Once CME 


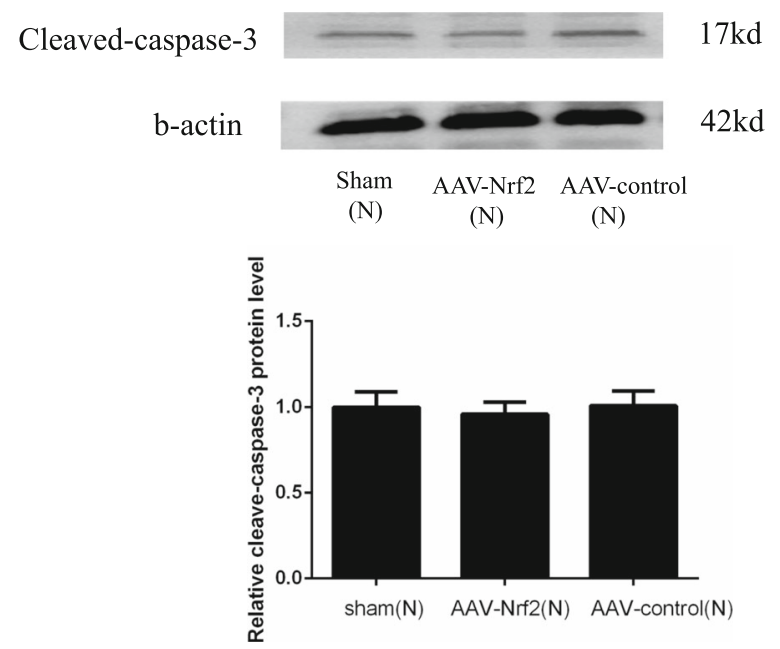

Fig. 6 The protein expression of cleave-caspase-3 in the groups of sham (N), AAV-Nrf2(N) and AAV-control (N) of which rats were not undergone operation. Nrf2: nuclear factor erythroid 2-like; AAV: adeno-associated virus. Data is shown as Mean \pm SD. \#: $P<0.05$ contrasted with sham $(\mathrm{N})$; *: $P<0.05$ contrasted with AAV-control (N)

happened, it cannot treat effectively [21]. CME gradually becomes a hot issue. More and more researches are focused on CME. The successful injection of plastic microspheres as the HE staining shown and the microinfraction of HBFP staining demonstrated the successful establishment of CME model. The CME model was the same as the previously persuasive studies $[22,23]$. We upregulated the expression of Nrf2 in rats by transfection of AAV-Nrf2 virus. In the preliminary experiment, the protein expression of Nrf2 in rats that were transfected was higher than the control rats $(P<0.05)$. The result meant the successful transfection of AAVNrf2 (Fig. 7).

In the present study, transfection of AAV-Nrf2 can deduce TUNEL-positive cells significantly. TUNEL assay data suggested that the upregulation of Nrf2 could inhibit CME-induced myocardial apoptosis clearly. LVEF, FS and $\mathrm{CO}$ of the AAV-Nrf2(CME) group were markedly increased, whereas LVEDd was reduced as contrasted with that of the CME group or AAV-control (CME) group significantly (Table 2). In comparison, the AAV-Nrf2(CME) group had a significant attenuation of levels of cTnI. It was also supported by a significant improvement in cardiac function of AAV-Nrf2(CME) rats.

Apoptosis is a major role in coronary microembolization [24]. The expression Bcl-2 and Bax, which belong to the Bcl-2 family members, is a key part in the cell apoptosis process. It reported that $\mathrm{Bcl}-2$ is an antiapoptotic protein and then $\mathrm{Bax}$ is a pro-apoptotic protein. The proportion of $\mathrm{Bcl}-2$ to $\mathrm{Bax}$ is considered as a crucial factor for ensuring the threshold of apoptosis [25]. In addition, it has been found caspase-3 is excited

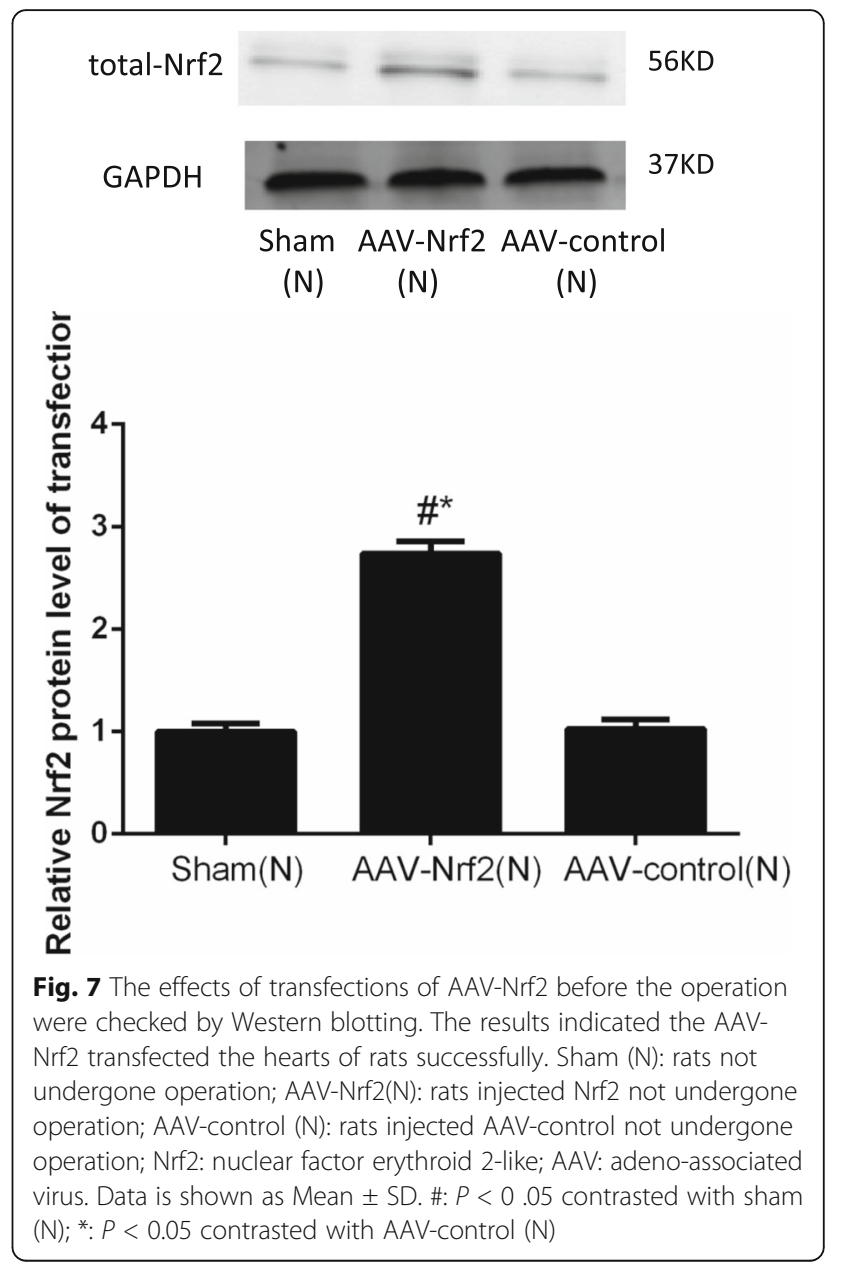

by the apoptotic approach and next processed into excited fragments like cleaved-caspase-3. It's thought to be an apoptotic coefficient [26]. Caspase-9 has the function to catalyze the activation of caspase- 3 [27, 28], whereas caspase- 9 is necessary in the cases of apoptotic cell mostly. A large number of studies have suggested that caspase-9 [29] and caspase-3 [30] are involved in the succession of myocardial infarction, also restriction of caspase-9 [31] and caspase-3 [32] can remarkably alleviate ischemia-induced myocardial apoptosis with heart function improvement. In this study, the expression of caspase3, caspase 9 and Bax/Bcl-2 significantly increased in CME and AAV-control (CME) groups, and was dramatically reduced in AAV-Nrf2 (CME) group (Figs. 4 and 5). These results indicated that transfection of Nrf2 could decrease the apoptosis of myocardial cells, and improve the cardiac function.

Nrf2 is a gene which has been identified as the especially vital part of Nrf2. The factor of Nrf2 can adjust regular evolving procedures, such as migration, cell growth, apoptosis [33] as well as invasion. Moreover, $\mathrm{Nrf} 2 / \mathrm{HO}-1$ signaling approach is a vital part in the 
process of apoptosis in the recent studies [34, 35]. Actually, Nrf2 is a crucial modulator of the expression of HO-1 [36].HO is a stress-responsive enzyme which could reduce highly deleterious free heme and produce anti-oxidants, anti-inflammatories, and regulators of apoptosis as well as multiplication, therefore maintaining homeostasis under pathological conditions [37, 38]. There are two distinct mammalian $\mathrm{HO}$ isoforms: $\mathrm{HO}-1$ and HO-2. HO-1 is upregulated in the myocardium of rats in response to ischemia-reperfusion injury. HO-1 can also protect cells from apoptosis induced by light [39]. Some researches have clarified that HO-1 expressing is adjusted primarily by Nrf2. Activated Nrf2 has been considered to be a crucial contributor to the expression of HO-1 [40]. Meanwhile, Ning et al. found that down-regulation of miR-142-5p attenuated ische$\mathrm{mia} /$ reperfusion injury in hippocampal neurons by promoting Nrf2/HO-1 signaling approach [41]. Liu et al. found that apoptosis was negatively correlated with the Nrf2 and HO-1 expressing, and ultimately affected the expression of cleaved caspase-3 [42]. In our study, mRNA and protein levels (Figs. 4 and 5) of Nrf2 and HO-1 upregulated in AAV-Nrf2(CME) rats and the Nrf2 could reduce the expression of caspase- 3 as well as caspase- 9 and a ratio of bax/bcl- 2 and apoptosis of myocardial cells (Fig. 3), and decrease levels of cTnI, demonstrating activation of Nrf2/HO-1 signaling can improve cardiac function in CME rats. The apoptotic pathways mainly include intrinsic and extrinsic pathways [43]. Some studies have confirmed that $\mathrm{Nrf} / \mathrm{HO}-1$ mainly regulated apoptosis through intrinsic pathway, but relative studies about extrinsic pathway of $\mathrm{Nrf} 2 / \mathrm{HO}-1$ were few $[44,45]$.In our study, Nrf2/HO-1 could suppress the expression of caspase- 9 and caspase- 3 , and caspase- 9 is the key factor of intrinsic pathway and active caspase- 3 to induce apoptosis. $\mathrm{Nrf} 2 / \mathrm{HO}-1$ is probably concluded in the process of myocardial apoptosis after CME through the intrinsic pathway. Whether Nrf2/HO-1 can regulate apoptosis through extrinsic pathway, further studies are still needed.

There are likewise some limitations in the current study: Firstly, the conclusions were drawn from the model of rat $\mathrm{CME}$ and it was prepared by the injection of the plastic microspheres into the LV. Thus, the present outcome might not be straightforward compared to the results achieved in the patient. Secondly, we only studied the short-term protection of Nrf2. Therefore, how long this protective effect lasts is uncertain. With this in mind, future research needs to be explored in more CME models.

\section{Conclusions}

These findings showed that activating Nrf2/HO-1 signaling can inhibit apoptosis to attenuate coronary microembolization, and also improve the cardiac function.
Nrf2/HO-1 signaling pathway is possibly a potential medical goal for treatment associated and it has a chance to be worthy for sick people who are undergone acute coronary syndrome or PCI and improve the longterm prognosis.

\begin{abstract}
Abbreviations
AAV: Adeno-associated virus; ANOVA: Analysis of variance; CME: Coronary microembolization; HO-1: Heme oxygenase-1; Nrf2: Nuclear factor erythroid 2-like; RT-PCR: Reverse Transcription-Polymerase Chain Reaction; TBST: Trisbuffered saline tween; TUNEL: TdT-mediated dUTP Nick-End labeling
\end{abstract}

\section{Acknowledgements}

We would like to thank Yuanxi Lu and Wenqin Guo for their technical support. In addition, we would like to thank all colleagues for contributing the experiments.

\section{Funding}

This research received support from National Natural Science Foundation of China (Grant No.81600283) and Guangxi Natural Science Foundation (Grant No. 2016GXNSFBA380022).

\section{Availability of data and materials \\ The unprocessed data from the current study can be obtained from the corresponding author.}

\section{Authors' contributions \\ $J L, L L$ and YS conceived the idea of the project. JL, XW, and QS conducted the experiments of real time-PCR and Western blotting and analyzed data. WH and YL collected samples. JL and LL wrote the manuscript. QS and YS participated in the revision of the important part of the manuscript. JL, XW and WG worked together to produce the tables and figures. All contributive authors of this original manuscript authorized the ultimate manuscript. All authors read and approved the final manuscript.}

\section{Ethics approval and consent to participate}

This experiment got the Ethics Committee of Guangxi Medical University, China permission and was carried out according with the Norms on the Animal Experiments. The animals were sampled and managed under the National Institute of Health (NIH Publication NO. 85-23, revised 1996).

\section{Consent for publication}

Not applicable.

\section{Competing interests}

All authors reach a consensus to consent for coming out in the journal. This paper has not been published elsewhere in whole or in part, which are no conflicts involved in the article.

\section{Publisher's Note}

Springer Nature remains neutral with regard to jurisdictional claims in published maps and institutional affiliations.

Received: 31 May 2017 Accepted: 13 October 2017

Published online: 24 October 2017

\section{References}

1. Erbel R, Heusch G. Coronary microembolization-its role in acute coronary syndromes and interventions. Herz. 1999;24(7):558-75.

2. Otto S, Seeber M, Fujita B, et al. Microembolization and myonecrosis during elective percutaneous coronary interventions in diabetic patients: an intracoronary Doppler ultrasound study with 2-year clinical follow-up. Basic Res Cardiol. 2012;107(5):1-12.

3. Heusch $G$, Kleinbongard P, Böse D, et al. Coronary microembolization. Circulation. 2009;120(18):1822-36

4. Falk E, Thuesen L. Pathology of coronary microembolisation and no reflow. Heart. 2003;89(9):983-5.

5. Breuckmann F, Nassenstein K, Bucher C, et al. Systematic analysis of functional and structural changes after coronary microembolization: a 
cardiac magnetic resonance imaging study. J Am Coll Cardiol Img. 2009; 2(2):121-30.

6. Dorge $H$, Neumann T, Behrends $M$, et al. Perfusion-contraction mismatch with coronary microvascular obstruction: role of inflammation. Am J Physiol. 2000;279(2):H2587-92.

7. Ferrari R, Balla C, Malagù M, et al. Reperfusion damage-a story of success, failure, and hope. Circ J. 2017:81(2):131-41.

8. Heusch G. The coronary circulation as a target of cardioprotection. Circ Res. 2016;118(10):1643-58.

9. Li L, Su Q, Wang Y, et al. Effect of atorvastatin (Lipitor) on myocardial apoptosis and caspase-8 activation following coronary microembolization. Cell Biochem Biophys. 2011;61 (2):399-406.

10. Liu T, Zhou Y, Wang JY, et al. Coronary microembolization induces cardiomyocyte apoptosis in swine by activating the LOX-1-dependent mitochondrial pathway and caspase-8-dependent pathway. J Cardiovasc Pharmacol Ther. 2016;21(2):209-18.

11. Liu T, Zhou Y, Liu YC, et al. Coronary Microembolization induces Cardiomyocyte apoptosis through the LOX-1-dependent endoplasmic reticulum stress pathway involving JNK/P38 MAPK. Can J Cardiol. 2015; 31(10):1272-81.

12. Jia $\mathrm{Z}$, Dong $\mathrm{A}$, Che $\mathrm{H}$, et al. 17-DMAG protects against hypoxia -/Reoxygenation-induced cell injury in HT22 cells through Akt/Nrf2/HO1 pathway. DNA Cell Biol. 2017;36(2):95-102.

13. Zhang JF, Su L, Ye Q, et al. Discovery of a novel Nrf2 inhibitor that induces apoptosis of human acute myeloid leukemia cells. Oncotarget. 2017;8(5):7625.

14. Chumboatong W, Thummayot S, Govitrapong P, et al. Neuroprotection of agomelatine against cerebral ischemia/reperfusion injury through an antiapoptotic pathway in rat. Neurochem Int. 2017;102:114-22.

15. Fu FY, Chen BY, Chen LL, et al. Improvement of the survival and therapeutic effects of implanted mesenchymal stem cells in a rat model of coronary microembolization by rosuvastatin treatment. Eur Rev Med Pharmacol Sci. 2016;20(11):2368-81.

16. Thielmann M, Dörge $H$, Martin C, et al. Myocardial dysfunction with coronary microembolization: signal transduction through a sequence of nitric oxide, tumor necrosis factor-al pha, and sphingosine. Circ Res. 2002;90(7):807-13.

17. Zhang QY, Ge JB, Chen JZ, et al. Mast cell contributes to cardiomyocyte apoptosis after coronary microembolization. J Histochem Cytochem. 2006; 54(5):515-23.

18. Hoggan MD, Blacklow NR, Rowe WP. Studies of small DNA viruses found in various adenovirus preparations: physical, biological, and immunological characteristics. Proc Natl Acad Sci. 1966;55(6):1467-74.

19. Atchison RW, Casto BC, Hammon WMD. Adenovirus-associated defective virus particles. Science. 1965;149(3685):754-5.

20. Zacchigna S, Zentilin L, Giacca M. Adeno-associated virus vectors as therapeutic and investigational tools in the cardiovascular system. Circ Res. 2014;114(11):1827-46.

21. Fu FY, Chen BY, Chen LL, et al. Improvement of the survival and therapeutic effects of implanted mesenchymal stem cells in a rat model of coronary microembolization by rosuvastatin treatment. Eur Rev Med Pharmacol Sci. 2016;20(11):2368

22. Wang X, Lu Y, Sun Y, et al. TAK-242 protects against apoptosis in coronary microembolization-induced myocardial injury in rats by suppressing TLR4/ NF-kB signaling pathway. Cell Physiol Biochem. 2017;41(4):1675-83.

23. Sun Y, Su Q, Li L, et al. MiR-486 regulates cardiomyocyte apoptosis by p53mediated BCL-2 associated mitochondrial apoptotic pathway. BMC Cardiovasc Disord. 2017;17(1):119.

24. Liu YC, Li L, Su Q, et al. Trimetazidine pretreatment inhibits myocardial apoptosis and improves cardiac function in a swine model of coronary microembolization. Cardiology. 2015;130(2):130-6.

25. Hu T, Wei G, Xi M, et al. Synergistic cardioprotective effects of Danshensu and hydroxysafflor yellow A against myocardial ischemia-reperfusion injury are mediated through the Akt/Nrf2/HO-1 pathway. Int J Mol Med. 2016; 38(1):83-94

26. Salvesen GS. Caspases and apoptosis. Essays Biochem. 2002:38:9-19.

27. Cain K, Bratton SB, Cohen GM. The Apaf-1 apoptosome: a large caspaseactivating complex. Biochimie. 2002;84(2):203-14

28. Rodriguez J, Lazebnik Y. Caspase-9 and APAF-1 form an active holoenzyme. Genes Dev. 1999:13(24):3179-84.

29. Takatani T, Takahashi K, Uozumi Y, et al. Taurine inhibits apoptosis by preventing formation of the Apaf-1/caspase-9 apoptosome. Am J Phys Cell Phys. 2004;287(4):C949-53.
30. Balsam LB, Kofidis T, Robbins RC. Caspase-3 inhibition preserves myocardial geometry and long-term function after infarction. J Surg Res. 2005;124(2): 194-200.

31. Sodhi RK, Singh M, Singh N, et al. Protective effects of caspase-9 and poly (ADP-ribose) polymerase inhibitors on ischemia-reperfusion-induced myocardial injury. Arch Pharm Res. 2009;32(7):1037-43.

32. Liu Q. Lentivirus mediated interference of Caspase-3 expression ameliorates the heart function on rats with acute myocardial infarction. Eur Rev Med Pharmacol Sci. 2014;18(13):1852-8.

33. Li P, Su L, Li X, et al. Remote limb ischemic postconditioning protects mouse brain against cerebral ischemia/reperfusion injury via upregulating expression of Nrf2, HO-1 and NQO-1 in mice. Int J Neurosci. 2016;126(6):552-9.

34. Lei $X$, Lei L, Zhang Z, et al. Neuroprotective effects of lycopene pretreatment on transient global cerebral ischemia-reperfusion in rats: the role of the Nrf2/ HO-1 signaling pathway. Mol Med Rep. 2016;13(1):412-8.

35. Zhang C, Wen C, Lin J, et al. Protective effect of pyrroloquinoline quinine on ultraviolet A irradiation-induced human dermal fibroblast senescence in vitro proceeds via the anti-apoptotic sirtuin 1/nuclear factor-derived erythroid 2related factor 2/heme oxygenase 1 pathway. Mol Med Rep. 2015;12(3):4382-8.

36. Sun GY, Li R, Cui J, et al. Withania somnifera and its withanolides attenuate oxidative and inflammatory responses and up-regulate antioxidant responses in BV-2 microglial cells. NeuroMolecular Med. 2016;18(3):241-52.

37. Fan J, Xu G, Jiang T, et al. Pharmacologic induction of Heme Oxygenase-1 plays a protective role in diabetic retinopathy in RatsHeme Oxygenase-1 in diabetic retinopathy in rats. Invest Ophthalmol Vis Sci. 2012;53(10):6541-56.

38. Soares MP, Bach FH. Heme oxygenase-1: from biology to therapeutic potential. Trends Mol Med. 2009;15(2):50-8.

39. Sun MH, Pang JHS, Chen SL, et al. Photoreceptor protection against light damage by AAV-mediated overexpression of heme oxygenase-1. Invest Ophthalmol Vis Sci. 2007;48(12):5699-707.

40. Zhang $M$, Wang $S$, Mao $L$, et al. Omega-3 fatty acids protect the brain against ischemic injury by activating Nrf2 and upregulating heme oxygenase 1. J Neurosci. 2014;34(5):1903-15.

41. Wang $N$, Zhang $L$, Lu Y, et al. Down-regulation of microRNA-142-5p attenuates oxygen-glucose deprivation and reoxygenation-induced neuron injury through up-regulating Nrf2/ARE signaling pathway. Biomed Pharmacother. 2017:89:1187-95.

42. Liu C, Vojnovic D, Kochevar IE, et al. UV-A irradiation activates Nrf2-regulated antioxidant defense and induces p53/Caspase3-dependent apoptosis in corneal endothelial CellsUV-A activates Nrf2 and induces p53 in corneal endothelial cells. Invest Ophthalmol Vis Sci. 2016;57(4):2319-27.

43. Hong S, Kim HY, Kim J, et al. Smad7 protein induces interferon regulatory factor 1-dependent transcriptional activation of caspase 8 to restore tumor necrosis factor-related apoptosis-inducing ligand (TRAIL)-mediated apoptosis. J Biol Chem. 2013;288(5):3560-70.

44. Zhang X, Liang D, Lian X, et al. Berberine activates Nrf2 nuclear translocation and inhibits apoptosis induced by high glucose in renal tubular epithelial cells through a phosphatidylinositol 3-kinase/Aktdependent mechanism. Apoptosis. 2016;21(6):721-36.

45. Yan B, Ma Z, Shi S, et al. Sulforaphane prevents bleomycin-induced pulmonary fibrosis in mice by inhibiting oxidative stress via nuclear factor erythroid 2-related factor-2 activation. Mol Med Rep. 2017;15(6):4005-14.

\section{Submit your next manuscript to BioMed Central and we will help you at every step:}

- We accept pre-submission inquiries

- Our selector tool helps you to find the most relevant journal

- We provide round the clock customer support

- Convenient online submission

- Thorough peer review

- Inclusion in PubMed and all major indexing services

- Maximum visibility for your research

Submit your manuscript at www.biomedcentral.com/submit
Biomed Central 